\title{
Actualidad en la etiología de la dislexia
}

\author{
Today in the etiology of dyslexia \\ Rosa Ma Rivas Torres y Santiago López Gómez \\ Dpto. Psicología Evolutiva y de la Educación Universidad de Santiago de Compostela
}

\begin{abstract}
Resumen
El estudio de las posibles causas de las dificultades lectoescritoras ha generado muchos conocimientos sobre su génesis, y se configura como un campo que todavía no está cerrado. En la etiología de las dislexias se han barajado factores genéticos, neurológicos, neurofisiológicos, cognitivos, madurativos, de personalidad, pedagógicos, etc. Sin embargo, resulta evidente la dificultad de poder identificar una única causa que las explique, ya que estos factores incluyen numerosas estructuras, procesos y funciones que, de modo interdependiente, contribuyen al dominio lectoescritor. Este hecho complica más su estudio. Abordamos el estado de la cuestión sobre la etiología de las dislexias. Para ello se revisan diferentes estudios considerando los diversos enfoques que mayor visibilidad tienen en la actualidad. Concretamente, se revisan los estudios neurológicos, los neurofisiológicos, los estudios cognitivos y los genéticos. Las aportaciones más notorias, si bien, lejos de ser concluyentes, tratan de demostrar que las conexiones entre las disfuncionalidades hemisféricas y las dificultades lecto-escritoras son cada vez más precisas y prometedoras.
\end{abstract}

Palabras clave: Dislexia. Etiología. Estudios neurológicos. Estudios neurofisiológicos. Estudios cognitivos. Estudios genéticos.

\section{Introducción}

La mayoría de los niños aprenden a leer rápidamente, una vez alcanzan un grado de maduración cognitivo determinado, en el marco de una enseñanza adecuada, de un medio familiar y de un contexto socioafectivo normalizados. No obstante, existen numerosos datos empíricos que reflejan que algunos niños inteligentes, capaces de realizar aprendizajes y resolver problemas complejos y con un desarrollo biopsicosocial que podríamos describir como normalizado, tienen serias dificultades para aprender a leer. Son niños con dificultades lecto-escritoras, también conocidos como disléxicos, con dislexia evolutiva o de desarrollo.

Puede afirmarse que existe una casi total unanimidad entre los autores que se dedican al estudio de la lectura al defender que ésta es una habilidad neurocognitiva compleja en la que intervienen gran cantidad de procesos. Unos son específicos de la propia habilidad lectora y que se describen como microprocesos o procesos de nivel bajo; perceptivos, de procesamiento subléxico y de acceso al léxico -p.e. identificación de letras, reconocimiento de palabras, manejo de las reglas de conversión grafema-fonema-. Otros son más generales; madurativos, cognitivos y de acceso al significado, descritos como macroprocesos o procesos de alto nivel -p.e. la atención y la memoria, la construcción del significado y la comprensión-; junto con algunos otros de naturaleza afectiva y motivacional -hábitos de lectura, deseo de aprender, seguridad y autoestima, autoconcepto académico- (Bravo, Villalón y Orellana, 2004; Weaver, 2002).

Las dificultades surgidas por déficits en el desarrollo y funcionamiento de estos procesos se manifiestan en alteraciones en la lectura, denominadas frecuentemente como dislexias. Se presentan como una dificultad, un retraso evidente o un fracaso con respecto al aprendizaje y dominio de la lectura de textos escritos y de las habilidades que requiere escribirlos. Son dificultades que se toman forma en el inicio del aprendizaje de la lectura y la escritura, sin causa aparente que las justifique, pues el niño cuenta con una instrucción convencional, una inteligencia adecuada, oportunidades socioculturales y ausencia de alteraciones orgánico-sensoriales.

\section{Etiología de la dislexia: revisión}

El estudio de las posibles causas de las dificultades lectoescritoras ha generado muchos conocimientos sobre su génesis. Nos encontramos con aportaciones de diferentes campos: neurología, psicología, lingüística,... y desde diversos modelos: psicolingüísticos, educativos, clínicos,... Dichas aportaciones han ofrecido múltiples explicaciones parciales o globales del hecho disléxico. Se han barajado factores genéticos, neurológicos, neurofisiológicos, cognitivos, madurativos, de personalidad, pedagógicos, etc. (Expósito, 2002; Gayán, 2001). Sin embargo, resulta evidente la dificultad de poder identificar una única causa que las explique, ya que estos factores incluyen numerosas estructuras, procesos y funciones que, de modo interdependiente, contribuyen al dominio lecto-escritor (Démonet, Taylor y Chaix, 2004).

Aun así, puede afirmarse que los resultados acerca de las conexiones entre disfuncionalidades hemisféricas y las dificultades lecto-escritoras son cada vez más precisos y prometedores (Galaburda y Cestnick, 2003).

Dentro de los estudios actuales que se dedican a investigar el origen de los problemas con la lectura sobresalen tres grandes líneas de trabajo, que en algunos estudios incluso llegan a converger, son: los estudios neurológicos, los neurofisiológicos, los cognitivos y los genéticos.

\section{a) Estudios neurológicos:}

Se han orientado hacia el rastreo de posibles alteraciones morfológicas entre los cerebros de sujetos disléxicos y normolectores a través, sobre todo, del empleo de técnicas de neuroimagen y del estudio de necropsias.

No existe un hallazgo concluyente y revelador sobre posibles anormalidades estructurales, si bien, se han identificado algunos factores relacionados con el funcionamiento cognitivo en las destrezas lectoras.

1. La falta de dominancia hemisférica, que podría explicar algunos tipos de errores que presentan los niños disléxicos al leer, como son los cambios de orientación o 
posición de letras y sílabas (p. e. peso - beso; sol - los) (Bakker, 1992, Bakker, Bouma, Gardien, 1990).

2. La menor tasa de procesamiento lingüístico por parte del hemisferio izquierdo, y que podría provocar una menor exactitud en la percepción de estímulos verbales (Lliadou, Kaprinis, Landylis y Kaprinis, 2010; Peterson y Pennington, 2012).

3. El funcionamiento deficitario del hemisferio derecho con interferencia de sus funciones sobre el izquierdo, lo que podría explicar algunas dificultades que muestran los sujetos disléxicos, como son algunos déficits en el procesamiento fonológico y en la velocidad de procesamiento visual (Facoetti, Turatto, Lorusso y Mascetti, 2001; Wolf y Bowers, 2000).

\section{b) Estudios neurofisiológicos:}

Muy relacionados con las investigaciones realizadas desde el paradigma neurológico destacamos los estudios neurofisiológicos. Desde la perspectiva neurofisiológica, sobre todo mediante el empleo de técnicas electromagnéticas (EEG, PEC, MEG) y de neuroimagen funcional (TEP, RMF), se ha tratado de describir cómo se produce la actividad cerebral mediante la realización de diferentes tareas, sobre todo de procesamiento fonológico.

Se ha puesto de manifiesto que en los sujetos disléxicos determinadas áreas no implicadas con tareas léxicas se sobreactivan, mientras que otras áreas con mayor implicación en tareas psicolingüísticas y de procesamiento fonológico muestran falta de activación al realizar tareas léxicas. Se evidencia, por tanto, un déficit en la integración neuronal de letras y sonidos (Blau, Atteveldt, Ekkebus, Goebel \& Blomert, 2009; Shaywitz et al., 2002).

\section{c) Estudios cognitivos:}

Se han estudiado los procesos cognitivos, sobre todo psicolingüísticos y, dentro de ellos, de manera específica, los de procesamiento fonológico, responsables de la lectura. Algunos estudios han evidenciado déficits de tipo cognitivo en los sujetos disléxicos. Entre los principales factores cognitivos constatados en sujetos con dificultades lecto-escritoras, se encuentran:

1. Los perceptomotrices -motricidad gruesa y fina, coordinación, percepción de formas- (Nicolson y Fawcett, 1994).

2. Los mnésicos, particularmente de memoria a corto plazo, a la hora de utilizar un código verbal fonológico (Martínez-Pérez, Majerus, Mahot y Poncelet, 2012; Ramus, 2001).

3. Los déficits en las habilidades de procesamiento fonológico, a la hora de representar y de manipular los componentes sonoros, la estructura fonológica del habla (Defior y Serrano, 2011; Ramus, 2001; 2003).

4. Los psicolingüísticos, responsables de déficits en la recepción, organización y expresión lecto-escritora (Nithart et al., 2009).

Muchos de los resultados encontrados al amparo de investigaciones que adoptan un enfoque neurológico o un enfoque más cognitivo no son del todo claros desde un punto de vista pragmático (Démonet et al., 2004). No obstante, el estudio de casos con descripciones de etiologías múltiples evidencia una gran variación en las dificultades lectoescritoras, lo que no significa que tal disparidad esté presente en todos los sujetos con estas dificultades (Galaburda y Cestnick, 2003; Ramus, 2001). De hecho, la singularidad de cada sujeto es habitual y, por ello, se dice que no hay una "dislexia", sino "disléxicos" tal y como, por otra parte, ocurre con casi todos los trastornos y dificultades.

\section{d) Estudios genéticos:}

Son estudios muy ligados a los anteriores, incluso confluyen en algunas de las ideas que afloran en los mismos, aunque tampoco han conseguido cerrar el debate sobre la etiología de la dislexia.

Los estudios genéticos y epidemiológicos defienden la existencia de una base genética en la dislexia e indican que la dificultad en la lectura puede heredarse en muchos casos.

Estos estudios han ampliado el panorama sobre diferentes genes que intervienen en la migración neuronal o en el aporte de alelos que condicionarían una mayor susceptibilidad frente a la dificultad lectora (Francks, MacPhie, \& Monaco, 2002; Williams \& O’Donovan, 2006). Ahora bien, dejan sin explicar un significativo porcentaje de casos que no responden, de forma específica, a dichos condicionantes genéticos.

Por todo ello, cabe resalta que nos encontramos en un momento de gran productividad en la investigación sobre la etiología de la dislexia. Aun así, y considerando algunas líneas como muy prometedoras, no se puede concluir que exista una explicación sobre el origen de la dislexia. No obstante, la actualidad dibuja un panorama en el que el abordaje multidisciplinar podrá acotar a corto plazo la génesis del trastorno lector.

\section{Referencias}

Bakker, D.J, Bouma, A, \& Gardien, C. (1990). Hemispherespecific treatment of dyslexia subtypes: a field experiment. Journal of Learning Disabilities, 2, 433-438.

Bakker, D.J. (1992). Neuropsychological classification and treatment of dyslexia. Journal of Learning Disabilities, 25, 102-109.

Blau, V., van Atteveldt, N., Ekkebus, M., Goebel, R. \& Blomert, L. (2009). Reduced neural integration of letters and speech sounds links phonological and reading deficits in adult dyslexia. Current Biology, 19(6), 503508.

Bravo, L., Villalón, M. y Orellana, E. (2004). Los procesos cognitivos y el aprendizaje de la lectura inicial: diferencias cognitivas entre buenos lectores y lectores deficientes. Estudios Pedagógicos, 30, 7-19.

Defior, S. y Serrano, F. (2011). Procesos fonológicos explícitos e implícitos, lectura y dislexia. Revista Neuropsicología, Neuropsiquiatría y Neurociencias, 11(1), 79-94.

Démonet, J.F., Taylor, M.J. y Chaix, Y. (2004). Developmental dislexia. The Lancet, 363, 1451-1460.

Expósito, J. (2002). Intervención educativa en la dislexia evolutiva: algunos aspectos psicobiológicos a considerar. Revista Complutense de Educación, 13(1), 185-210.

Facoetti, A., Turatto, M., Lorusso, M.L. \& Mascetti, G.G. (2001). Orienting of visual attention in dyslexia: evidence for a asymmetric hemispheric control of attention. Experimental Brain Reseach, 138, 46-53.

Francks, C., MacPhie, I.L. \& Monaco, A.P. (2002). The genetic basis of dyslexia. Lancet Neurology, 1(8), 483490.

Galaburda, A. y Cestnick, L. (2003). Dislexia del desarrollo. Revista de Neurología, 36(Sup.1), S3-S9.

Gayán, J. (2001). La evolución del estudio de la dislexia. Anuario de Psicología, 32(1), 3-30.

Lliadou, V., Kaprinis, S., Kandylis, D. \& Kaprinis, G.S. (2010). Hemispheric laterality assessment with dichotic digits testing in dislexia and auditory processing 
disorders. International Journal of Audiology, 49, 247252.

Martínez-Perez, T., Majerus, S., Mahot, A., \& Poncelet, M (2012). Evidence for a specific impairment of serial order short-term memory in dyslexic children. Dyslexia, 18(2), 94-109.

Nicolson, R.I., y Fawcett, A.J. (1994). Comparison of deficits in cognitive and motor skills among children with dyslexia. Annals of Dyslexia, 44, 147-164.

Nithart, C., Demont, E., Majerus, S., Leybaert, J. Poncelet, M., y Metz-Lutz, M.N. (2009) Reading disabilities in SLI and dyslexia result from distinct phonological impairments. Develomental Neuropsychology, 34, 296311.

Peterson, R.L. \& Pennington, B.F. (2012). Developmental dyslexia. The Lancet, 26(379), 1997-2007.

Ramus F. (2001). Outstanding questions about phonological processing in dyslexia. Dyslexia, 7, 197-216.
Ramus, F. (2003). Developmental dyslexia: specific phonological deficit or general sensorimotor dysfunction? Current Opinion in Neurobiology, 13(2), 212-218.

Shaywitz, S.E., Shaywitz, B.A., Pugh, K.R, Mencl, W.E., Fulbright, R.K., Skudlarski, P., et al. (2002). Disruption of posterior brain systems for reading in children with developmental dyslexia. Biological Psychiatry, 52, 101110.

Weaver, C. (2002). Reading process and practice. Portsmouth: Heinemann.

Williams, J. \& O’Donovan, M.C. (2006). The genetics of developmental dyslexia. European Journal of Human Genetics, 14, 681-689.

Wolf, M. \& Bowers, P.G. (2000). Naming-Speed processes and developmental Reading disabilities: An introduction to the special issue on the Double-Deficit hypothesis. Journal of Learning Disabilities, 33, 322-324. 